\title{
A game for robot operation training in Search and Rescue missions
}

\author{
R. Gonçalves ${ }^{1,2}$, R. Baptista1, ${ }^{1,}$, A. Coelho1,2, A. Matos ${ }^{3}$, C. Vaz de Carvalho ${ }^{3,4}$, J. Bedkowski $^{5}$, P. \\ Musialik $^{5}$, I. Ostrowski ${ }^{5}$, K. Majek ${ }^{5}$ \\ ${ }^{1}$ INESC/USIG, Porto, Portugal \\ ${ }^{2}$ Faculty of Engineering of University of Porto /DEI, Porto, Portugal \\ ${ }^{3}$ Faculty of Engineering of University of Porto /DEEC, Porto, Portugal \\ ${ }^{4}$ School of Engineering, Polytechnic of Porto, Portugal \\ ${ }^{5}$ Institute of Mathematical Machines IMM, Poland
}

\begin{abstract}
Search and rescue (SAR) teams often face several complex and dangerous tasks, which could be aided by unmanned robotic vehicles (UV). UV agents can potentially be used to decrease the risk in the loss of lives both of the rescuers and victims and aid in the search and transportation of survivors and in the removal of debris in a catastrophe scenario. Depending on the nature of a catastrophe and its geographical location, there are potentially three types of UVs that can be deployed: aerial, surface and ground. Due to the control and manipulation particularities each type of UV contemplates, their operators need prior training and certification. To train and certify the operators a tool (serious games) is under development. In this paper we will make an overview about our approach in its development. This game uses a typical client-server architecture where all client agents (virtual UVs and operator client interfaces) share the same immersive virtual environment which is generated through the merging of GIS data and a semantic model extracted from 3D laser data. There will be several types of scenarios suitable to several types of catastrophe situations. Each of these scenarios has its own mission plan for the trainees to follow. The game will also provide an interface for mission planning so that each mission plan will be carefully designed to accurately correspond to a matrix of skills. This matrix lists a set of common skills in various different $U V$ operational case studies which will allow the certification of operators.
\end{abstract}

Index Terms-Certification, robots, Serious games, simulation, training

\section{INTRODUCTION}

In light of recent disasters, such as the earthquakes in Haiti and Japan, it is becoming more apparent that search and rescue (SAR) teams could gain a substantial benefit from the use of unmanned vehicles (UV) to aid them with some of the most life threatening challenges faced in the field. These tools can help SAR teams remove debris in a faster and safer manner; be able to access and get overviews of areas and victims that are inaccessible to humans; reinforce building structural integrity; provide medical aid to victims and transportation; assess geographical areas and create intervention plans; etc. By actively helping in all these activities, UVs may provide a great contribution to the celerity in finding and rescuing victims. However, because these UVs aren't totally autonomous, their contribution to the SAR teams will be influenced by the controllers' UV operating skills. This is why having tools capable of training and certifying operators in the use of UVs is necessary.

This paper presents a general overview of a serious game (SG) based training system architecture, currently in early stages of development, composed by two main components: scenario/operation builder and a SG for UV training. The general process of how the scenarios and missions are created is also explored as well as the methods used to assess and certify UV operator trainees. This system aims to train and certify operators in three types of UV:

- Unmanned aerial vehicle (UAV) - Although these vehicles are mostly autonomous, some operations such as the landing on surface vehicles are still done by human operation.

- Unmanned surface vehicle (USV) - Operators can interact with these types of vehicles by sending high or low level commands.

- Unmanned ground vehicle (UGV) - These vehicles can be navigated either autonomously or by human operation. Some of them may also have a robotic arm which is also controlled by an operator.

This paper is organized as follows. Section II presents the motivation and some important definitions. In section III some related work is explored, followed by a general description of the overall system architecture in Section IV. Section V further details the UV game trainer component, focusing on more specific aspects such as mission definition. Section VI explains the how can UV operator certification be achieved. Finally, in section VII, a small case study is presented followed by section VII with the conclusions and an outline of future work.

\section{GAMES FOR TRAINING}

Serious games are becoming increasingly more popular in the corporate level as well as in the research community. In spite of all this attention there is still a lot of different definitions of what a serious game is. For this paper SGs are defined as follows: Serious games are applications which aim to take advantage of all the features that make games fun and engaging and use them to empower learning [1], promoting trainees' interest by making the focused educational subject exciting. 
The system presented on this paper intends to simulate UVs operations with a high degree of fidelity and for this system to be classified as a serious game it needs to incorporate formal structural elements that are common in games such as rules, choices, cooperation, competition, trial-and-error, etc [2].

Training is defined as an organized activity aimed at imparting information and/or instructions to improve the recipient's performance or to help him or her attain a required level of knowledge or skill ${ }^{1}$. Using games and structured learning activities in training are a delightful way to bring key topic areas to your learner [3]. On Serious games taxonomy defined by Ben Sawyer and Peter Smith, the games for training fits in several areas as Government, Defense, education, corporate and industry which different aspects as occupational safety, skills, communications, and orientation. Several examples of games for these aspects are Alcoa SafeDock, Rosser Surgery Skills w/Games, Shield of Freedom - a training simulator for the U.S. Coast Guard and America's Army.

\section{RELATED WORK}

Besides the serious game element present in this work, there is also a strong and extremely important unmanned vehicle simulation aspect. At the present moment several tools exist to simulate and visualize UVs operating since this type of software not only allows a high potential decrease of resource costs, but also provides a platform for safer and faster testing. One of such applications is the USARSim [4], an open source framework built on top of the Unreal Engine to simulate multi-robots and environments. In addition, interfacing with the Mobility Open Architecture Simulation and Tools framework $(\mathrm{MOAST})^{2}$ which provides a modular control system of robot control and customization, allows the system users to add their own modules or alter existing ones in order to obtain more complex robots than USARSim is capable of implementing.

Another example of such tools is the Webots [5] which also allows some degree of robot customization at the shape and attributes level. This tool also has the advantage of allowing robots to be independent from the tool and communicate with it remotely through TCP/IP. To handle physics simulation Webots uses Open Dynamics Engine (ODE) ${ }^{3}$, an open source library.

The previous examples are mostly pure simulation tools, however there are others that were developed with real and specific vehicles in mind. One of such tools is the AUV Workbench [6]. This tool supports the creation of real-time task-level missions, their rehearsal and visualization as well as replay. Visualization can be done in $2 \mathrm{D}$ or $3 \mathrm{D}$, the latter being possible through VRML technology. In both cases the environments are built through GIS data composition for maximum fidelity of the real environments where missions will occur, either simulated or live. After creating a mission, users have the option of saving it into XML for later use.

\footnotetext{
${ }^{1} \mathrm{http}: / / \mathrm{www} \cdot$ businessdictionary.com/definition/training.html

$2 \mathrm{http}: / /$ moast.sourceforge.net

3 http://www.ode.org
}

Another tool that can be classified in this category is the Neptus [7], a command and control framework. This tool uses a modular approach composed of the following components: the mission console, responsible for executing missions and interfacing with the other modules; the mission planner, responsible for mission setup and preparation which includes a user friendly interface and map generation; the mission reviewer, responsible for gathering data during mission execution and supports the replay of past missions for analysis; the mission data broker, responsible for managing data and providing web services to access that data; and finally the vehicle simulator, responsible for simulating real vehicles concurrently for mission previews.

All these examples have peculiarities that will prove useful for our own approach, such as possibility of having the UV's separate from the core framework in such a way that the vehicles used in a mission can be either simulated or real, or in other words, support of hardware in the simulation loop; or having a complete and user friendly interface to plan and deploy missions. By combining some of these elements with a game oriented approach, we hope to achieve a balance between both worlds and at the same time provide a more appealing alternative for learning how to operate these systems.

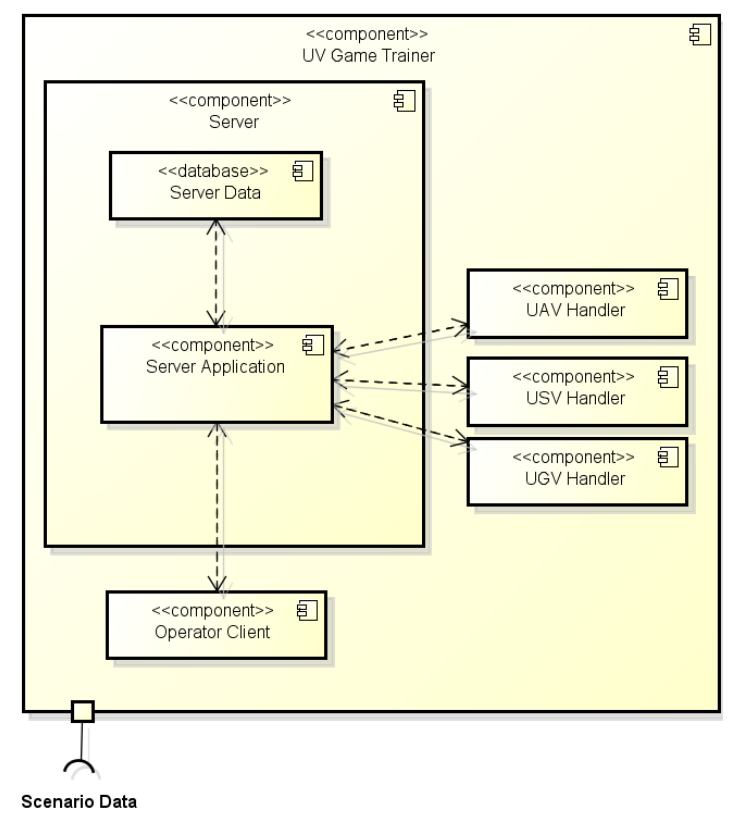

Figure 1. UV Game trainer system architecture

\section{SyStEM ARCHITECTURE}

The game based training system for UV operational training is composed by two main components. Firstly is the UV training system (Fig. 1) and secondly is the scenario and operation creator application (Fig. 2).

The UV training system, as the name suggests, is the system with which trainees interact to train and learn how to operate UVs as well as understand their operational particularities and how to cooperate with other operators. This system is composed by three sub components which 
are connected through networking, either LAN or WAN. At the core of this training system lies the server. Its main objective is the coordination between the $3 \mathrm{D}$ game client application which is the interface used by the trainees to interact with the system, the simulated vehicles and the game logic/missions. The server is non-authoritative towards the UV simulation components since they operate as extensions to the server's logic and decide what the UVs can or cannot do. However towards the client the server is full-authoritative to avoid circumventing the game's logic, in other words, to prevent any kind of cheating.

Each UV has its own simulation component which communicates with the server, through a network, receiving input from the client component and returning operation results and vehicle status that includes its position and orientation among other important information. The network communication between the server and the UVs simulators allows their placement in dedicated computers, instead of having the server and its several UVs sharing the same resource pool.

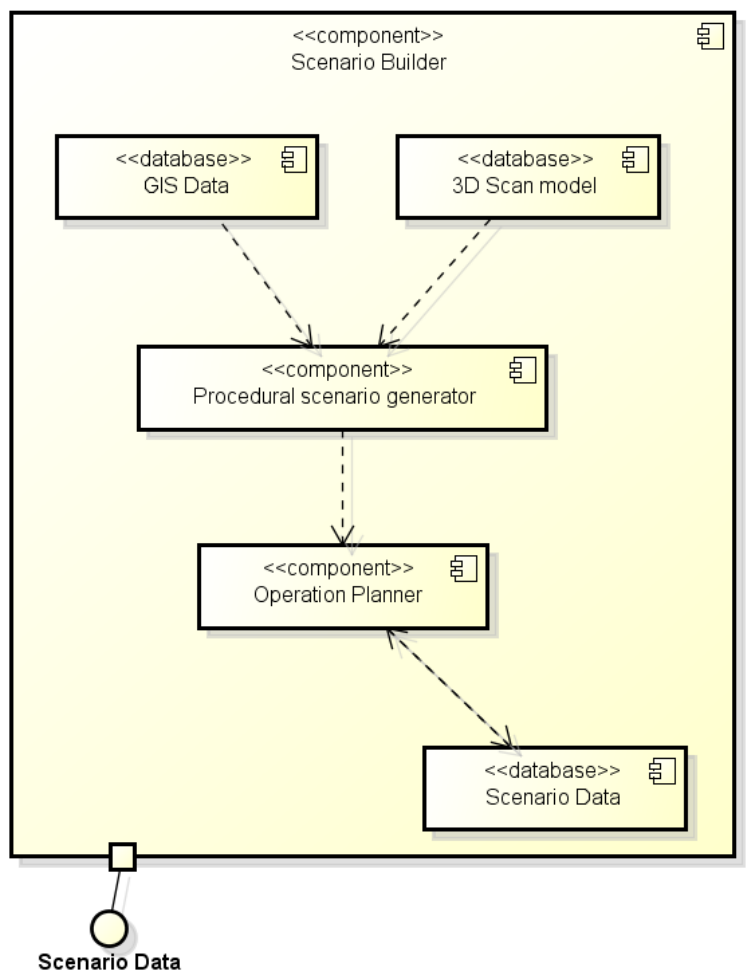

Figure 2. Scenario and operation builder architecture

The scenario and operation creator component is composed by two main components and three data sources. The first component, scenario generator, is responsible for creating the scenarios in which operators will train and it's composed mainly of procedural generation processes'. The second component is a tool that reads the generated scenarios and provides a set of tools that allows the system administrators to create operation specifications and save it to a database for later re-use or tweaking, as well as export both the scenario and associated operations to the UV training system component.
The scenario data exported by the Mission Planner component is comprised of a 3D model of the training environment generated by the Scenario creator component; mission relevant dynamic objects, such as docks or debris that have to be removed, etc; UV information such as deployment coordinates and initial definition of mission parameters; tutorial information and finally operation specification. All coordinates are associated with the generated scenario.

\section{UV GAME TRAINER}

Currently most of the modules that compose the overall system architecture are still in early stages of development except for the UV Game trainer component which has already been used to simulate a potential high sea operation scenario, further explained in section VII. Even though the scenario and operation builder module is still in early stages of development, the creation of high sea missions is already possible due to the fact that this type of mission needs for procedural map generation data is extremely low and therefore we are able, for the moment, to bypass the procedural scenario generator module and roughly plan and deploy operations of this type.

As mentioned in the previous section, the server application is the core of the UV Game trainer component and some of its main functions are receiving inputs from the operator client application, managing information and feeding data to each connected UV. Some of the input data sent from the operator are actual mission execution plans that are then processed by the server. These mission plans are coded in XML format, as seen in Fig.3 example, and are generally composed of task information and to which UV they are assigned to. Tasks are groups of maneuvers and other UV specific operations such as turning on sonar and retrieve data or other complex hardcoded maneuvers such as expansive square search. The mission execution plans created by learners are one of the ways they have to interact with the system and complete the assigned operation.

An operation is a set of timed objectives for one or more UVs to be done either in cooperation or solo. If an operation is solo each UV operator will train independently from other operators, however if they are cooperative they may require two or more objectives to be accomplished in parallel in order to advance. Each objective has a minimum time in which it can be executed and a score. The objective score is influenced by time in the sense that after it's successfully executed, depending on the difference between the minimum time and the actual time the operator took to accomplish the task, the score will change accordingly. Associated with these scores there will be a table of high scores, motivating operators to train more in order to obtain a better score and consequently a better position in the table. This will also give them a sense of progress and achievement, further motivating them. The cooperative element should also promote inter-help between the operators, by sharing information and knowledge in order to achieve better results as a team. 
This system will also provide tutorial support so that trainees can be given the possibility to access, at any point of the training, extra pedagogic information that may aid them both in getting around with interface and the UVs and their usage. There are two types of tutorials: operation tutorials where an operation is totally dedicated as form of basic optional information and the second type is added information for operation missions. Either type of tutorials may be associated with mission objectives so that when a trainee starts a certain objective, the system automatically (if tutorials are activated) start a tutorial related with the new task. If a trainee is repeating a mission, and is therefore familiar with it, he may disable tutorials so that he can move faster to the training instead of having to replay the tutorials.

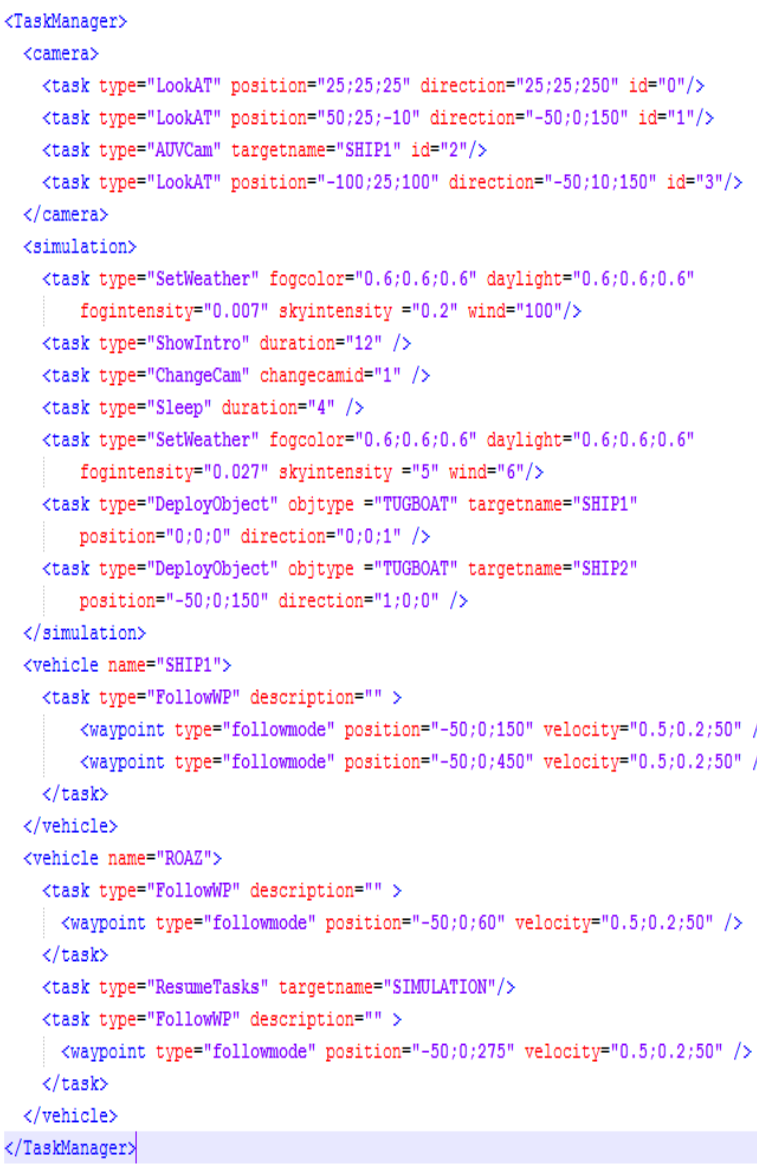

Figure 3. XML partial example of a mission execution plan for cinematic view

Another feature of this system is the cinematic replay ability of operations which can be used, for instance, to display to an audience how a type of procedures present during the execution of specific types of operation missions and vehicle maneuver capabilities. This is why the complete XML mission plan contains an object camera which enables the application to chance the main camera position or switch between vehicles' mounted cameras.

Another important aspect of the game is its graphical quality and fidelity which are very important aspects for player intrinsic motivation [7][8] as well as immersion.
With that in mind, one of these project goals is to achieve a high quality experience, both visual and audio wise.

\section{CERTIFICATION}

This paper presents the general system architecture for a serious game which main objective is to train and certify operators in the use of different unmanned vehicles. This system is being developed with the intent of using real scenarios, generated from GIS data and 3D laser scanning. This way the training will be more realistic and operators will benefit from knowing the general layout of the location the UVs will operate, therefore being better prepared.

The competences training is a structured approach to training and assessment that is directed towards achieving specific outcomes. Based on this approach, the certification is possible and assures the quality of training.

The use of different unmanned vehicles represents a several types of competencies, which users needed to be individual's abilities to make deliberate in contextdependent and imply integration of knowledge, skills, judgment and attitudes [10]. These types can be personal, functional or task-oriented, where firstly, the personal competencies represent the core of knowledge, skill and attitude that each person should have for superior performance. The second type is functional competency, which is dependent of a particular area or profession and is related to technical knowledge or required skills. Lastly, the task competencies are implicit and associated with specific role functions.

This process of certification of competences can be defined in two distinct phases. The first is the analysis of all the competences required to perform any given task/mission. For that, it's necessary to determine the competency model, where it is categorized what core skills are needed to be successful in any work environment or "a collection of competencies associated with a successful performance" [11]. The organization of competences can be performed in a variety of modes and one of them is the competency profiling. It's defined as a set of competencies and includes associated behaviors that link directly to the work to be performed, as well as the levels of proficiency for each behavior. Typically there are several competencies (5-10) for any specific task. Through valid, fair and unbiased competency profiles, the individual training performance can be analyzed and measured for strengths and weaknesses.

The second phase of certification is assessing competency. This part is a form of assessment that is driven from a specific set of outcomes, general and specific, and contributes to make judgments with respect to learners' achievements and non-achievements of these outcomes. Evaluation and certification are then obtained through these outcomes based on the demonstrated achievements. The focus of this process is based on actual skills and knowledge that a learner demonstrates on a specific task, verifying that he possess the competency and it's applicable to task. 
This last phase should be defined on 5 steps as shown in Fig.4. The first step is competences self-assessment by the learner/user. Based on the identified skills and knowledge, performance data is collected to be used on the next step. The second step, data analysis, is associated with verifying the data in order to make qualitative reviews. This task can be performed with or without supervisor.

The third step is the identification of the learner needs based on the necessity of the skills and knowledge and suggesting possible training subjects. The fourth step is the training of the identified skill and knowledge necessities, both in and outside of the work environment. Lastly, after finishing the training period, the reassessment follows in order to find the new competence level. By comparing the initial competence level to the new one, an evaluation decision can be made.

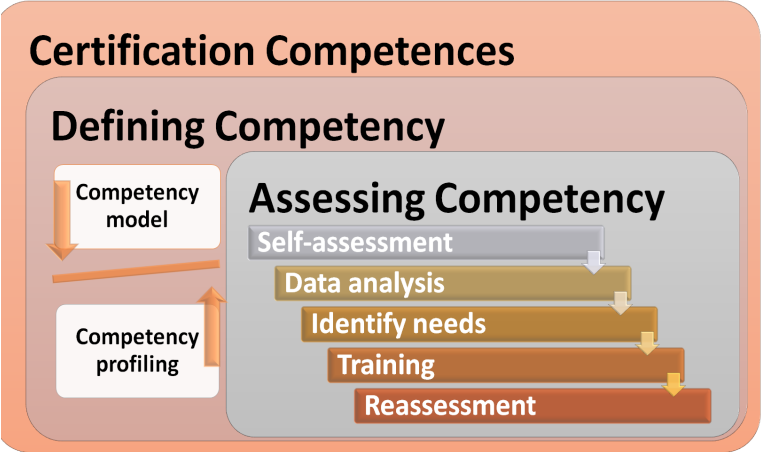

Fig. 4 The competency certification process

This certification architecture is intended to help the development the serious games to certify training competences according the defined missions. The first step of this process is analyzed situations and scenarios of training/certification. The definition of a useful scenario of certification is the aggregation of contextual or environmental different activities and tasks representative of skills, abilities or knowledge needed to perform and succeed in real situations. After this step, the competences profiles of the target group must be defined, as well as, that must be used to obtain the correct outcomes of each mission/scenario. This interactive process between competency (goals, activities and proficiency levels) and scenarios (goals, situations and outcomes) is a very useful exercise to obtain both the competences profile and several ideas to develop game.

This in-game certification component can be developed based on methodology for in-game certification [12] to build a correlation matrix between competencies and game mechanics. This tool is build based on a case study to support the game development.

The in game certification aspect is also focused and is a key feature of the system which means it requires the integration of methodologies that will enable the assessment of the trainees progress according to the defined mission objectives. Besides these methodologies, the tools for mission and scenario design will also enable the creation of missions that are as close and accurate to real world missions.

\section{CASE STUDY}

As mentioned on chapter $\mathrm{V}$, a operation simulation has been created. This simulated operation takes place at high-sea where weather conditions (dense fog) prevent good visibility (Fig. 5), which lead to the collision between two ships. In consequence of the collision both ships sink and their passengers were forced to jump overboard, however still having time to send a distress signal.

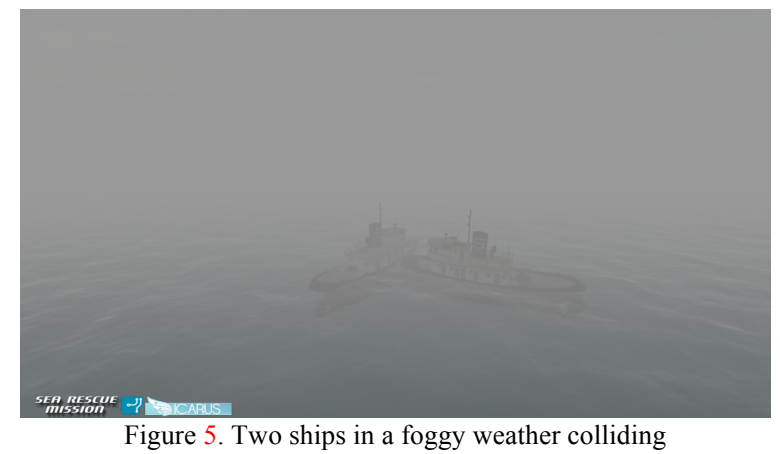

After receiving the distress signal, the local SAR agency immediately launched a rescue operation and deployed an autonomous surface vehicle to the calculated area with highest probability of finding survivors. As the SUV (Fig. 6) reaches the designated area it begins a grid search of the area and as soon as it finds survivors it launches a smaller vessel equipped with an inflatable raft, ending this simulation with the survivors swimming towards the raft and inflating it.

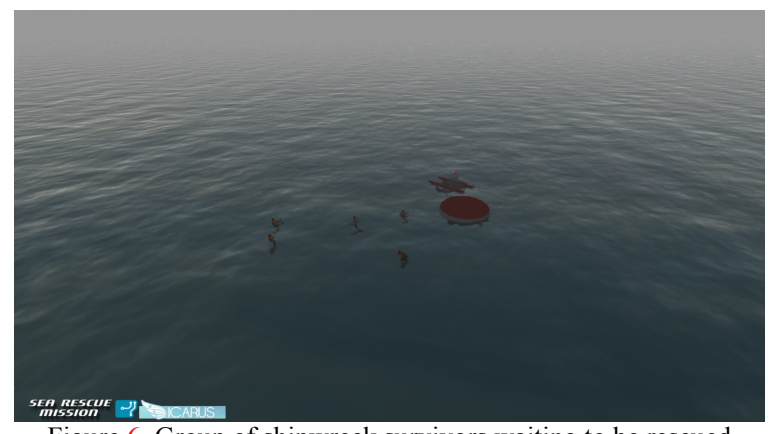

Figure 6. Group of shipwreck survivors waiting to be rescued

\section{CONCLUSIONS AND FUTURE WORK}

This platform for teaching operators how to work with different types of unmanned vehicles is still under development. This platform is an attempt of bridging various subjects under the same framework, simulation; digital games and certification. We believe by achieving balance between all three elements we can offer a valuable alternative to trainers and trainees, and, thanks to the tests we performed, as shown in chapter VII, we believe we are on the right track, even though that case study only depicts one part of the whole. 
For the moment vehicles are still being simulated within the server, however we are already have a prototype module where vehicles are operating remotely and it's near the point of integration into the framework. Map generation is also underway by extracting data from sources such as OpenStreetMap and combine it with point cloud data for higher fidelity environments.

\section{ACKNOWLEDGMENT}

The work presented in this paper is being funded by the European Community's Seventh Framework Program (FP7/2007-2013) under grant agreement n285417

\section{REFERENCES}

[1] T. Susi, M. Johannesson and P. Backlund. "Serious games: An overview", 2007.

[2] M. Prensky, "Simulations: Are They Games?", Digital gamebased learning, 2001.

[3] S. Sugar, and J. Whitcomb. Simple and Effective Techniques to Engage and Motivate Learners. ASTD, 2006.

[4] S. Carpin, M. Lewis, J. Wang, S. Balakirsky and C. Scrapper. USARSim: a robot simulator for research and education. In Robotics and Automation, 2007 IEEE International Conference on (pp. 1400-1405). IEEE. (2007, April)

[5] O. Michel. WebotsTM: Professional mobile robot simulation, 2004. arXiv preprint cs/0412052.

[6] C. Lee. NPS AUV Workbench: Collaborative Environment for Autonomous Underwater Vehicles (AUV) Mission Planning and 3D Visualization. Naval Postgraduate School Monterey CA, 2004.

[7] A. Wigfield, J. Guthrie, S. Tonks and K. Perencevich. Children's motivation for reading: Domain specificity and instructional influences. Journal of Educational Research, 97, pp. 299-309, 2004.

[8] B. Patrick, H. Jennifer and T. Kempler ."What's everybody so excited about?". The effects of teacher enthusiasm on student intrinsic motivation and vitality. The Journal of Experimental Education 68.3, pp. 217-236, 2000.

[9] P. Dias, G. Goncalves, R. Gomes, J. Sousa, J. Pinto and F. Pereira. Mission planning and specification in the Neptus framework. In
Robotics and Automation, 2006. ICRA 2006. Proceedings 2006 IEEE International Conference on (pp. 3220-3225). IEEE, 2006.

[10] Govaerts, M., Educational competencies or education for professional competence?. Medical Education. 42, pp. 234-236, 2008.

[11] Garman, A. and Johnson, M. Leadership competences: An introduction, Journal of Healthcare Management, 51(1), pp.13-17, 2006.

[12] R. Baptista, A. Coelho and C. V. Carvalho. Methodology For InGame Certification In Serious Games, Proceedings EDULEARN13, pp. 3152-3162, 2013.

\section{AUTHORS}

R. Gonçalves is with USIG, INESC - TEC, Rua Dr Roberto Frias 378, 4200-465 Porto (e-mail: refg@fe.up.pt).

R. Baptista is with USIG, INESC - TEC, Rua Dr Roberto Frias 378, 4200-465 Porto (e-mail: rjvb@inescporto.pt).

A. Coelho is with USIG, INESC - TEC, Rua Dr Roberto Frias 378, 4200-465 Porto (e-mail: acoelho@fe.up.pt).

A. Matos is with ROBIS, INESC - TEC, Rua Dr Roberto Frias 378, 4200-465 Porto (e-mail: anibal@fe.up.pt).

C. Vaz de Carvalho is with ISEP, Rua Dr. António Bernardino de Almeida431, 4200-072 Porto (e-mail: cmc@isep.ipp.pt).

J. Bedkowski is with IMM, ul. Ludwika Krzywickiego 34, 02-078 Warsaw (e-mail: januszbedkowski@gmail.com).

P. Musialik is with IMM, ul. Ludwika Krzywickiego 34, 02-078 Warsaw (e-mail: pawelmus@op.pl).

I. Ostrowski is with IMM ul. Ludwika Krzywickiego 34, 02-078 Warsaw (e-mail: iostrowski@wp.pl).

K. Majek is with IMM, ul. Ludwika Krzywickiego 34, 02-078 Warsaw (e-mail: karolmajek@gmail.com). 